\title{
Telling apart motor noise from exploratory behaviour, in early development
}

\author{
Teodora Gliga
}

Centre for Brain and Cognitive Development, Birkbeck, University of London

\begin{abstract}
Infant's minutes long babbling bouts or energetic reaching for or mouthing of whatever they can get their hands on gives very much the impression of active exploration, a building block for early learning. But how can we tell active exploration from the activity of an immature motor system, attempting but failing to achieve goal directed behaviour? I will focus here on evidence that infants actively increase motor activity and variability when faced with opportunities to gather new information. I will discuss mechanisms generating movement variability, and suggests that, in the various forms it takes, from deliberate hypothesis testing to increasing environmental variability, it could be exploited for learning. However, understanding how infant exploratory behavior contributes to learning will require more in-depth investigations of both the nature of and the contextual modulation of behavioural variability.
\end{abstract}


Parents easily tell when a child is eating and when she is only playing with her food. In this latter case, she would be squashing her potatoes, sliding her fingers through the mash, mixing it with water, dropping it on the floor (repeatedly) and occasionally, mouthing it, only to spit it seconds later. They also recognize as play their infant's minutes long babbling bouts or energetic reaching for or mouthing of whatever they can get their hands on. Parents, just as psychologists, have an ambivalent attitude towards this behaviour, appreciating the joy it brings and suspecting it must be an opportunity for learning while also puzzling at the sheer amount of time infants spend engaged in, what looks like, endless variation and repetition of nonsensical actions. Are parents, like myself, right in perceiving the above the above behaviors as play (Fisher et al, 2008)? The problem researchers face in answering this question is that variability in motor outputs, which may look like exploratory behaviour, has also often been described as the manifestation of an immature system, attempting but failing to achieve goal directed behaviour (e.g. Yan et al., 2000). In the skill acquisition literature movement variability is an index of error or noise in sensory-motor systems, something an organism strives to eliminate (Harris \& Wolpert, 1998; Todorov \& Jordan, 2002). Gibson (1988) had already signalled this conundrum, when describing infant behavior. While suggesting that "The active obtaining of information that results from the spontaneous actions of the infant is a kind of learning", she also raised the question of whether "this activity is in any way controlled by the infant", rather than "compulsory response to stimulation".

Some have made the further distinction between exploration -aimed at discovering an object's function, for example - and play -aimed at discovering what one can do with a particular object (Hughes, 1978). How this distinction is operationalized is less than clear cut - when learning is not guided by ostensive teaching, an object's function is whatever a child can do with it (Bonawitz et al, 2011). In addition, not all authors adhere to this distinction. 
Singer \& Singer, 2005 play represents a natural way for children to explore and learn about themselves and the world about them. Whether we have in mind behaviour that is exploration or play, the same principles apply, e.g. the higher attention given to means rather than the ends (Ferguson \& Macken, 1983), often reflected in increased variation in behaviour, where movements are exaggerated, action sequences are reordered or incomplete, but also often repeated (Rubin et al, 1983). The same question of whether this behavior is actively produced to serve learning, therefore applies to both play and exploration. However, since most work relevant to this question has concerned behaviors that would more readily be described as exploration, I will refer to it as such.

My goal here is to provide evidence that, from very early in life, infant behaviour is driven to gaining information and can, therefore, be referred to as active exploration. I will first focus on evidence that motor activity and variability can be upregulated to serve learning. Given the aim of this review is to illustrate the above principle, evidence will not exclusively come from human development. I will describe (1) developmental changes in the amount and variability of motor activity, when the growing organism faces new learning opportunities; (2) decreased and not increased variability in certain developmental disorders; (3) an increase in in the amount and variability of motor activity with information availability and finally, and, finally, (4) direct links between in the amount and diversity of motor activity (henceforth exploratory activity) and learning. I will then move on to discussing the mechanisms that could generate exploratory behaviour, focussing especially on how variable movement (see Figure 1) can be generated, and those linking variability to learning. I will discuss which of these mechanisms could be at play in early development. 


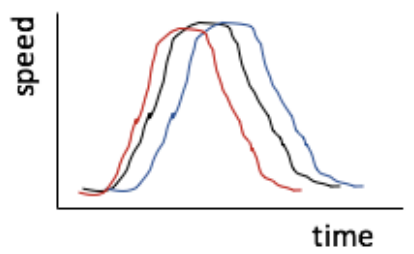

VARIATION IN ACCELERATION

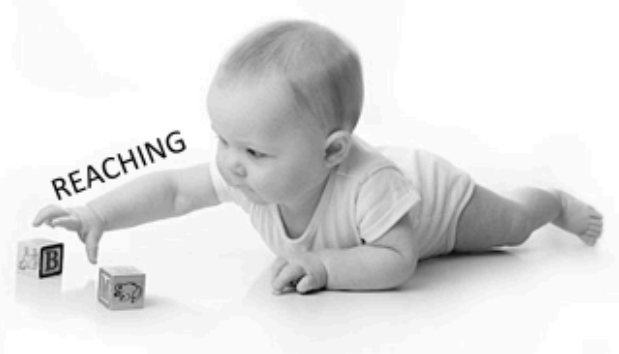

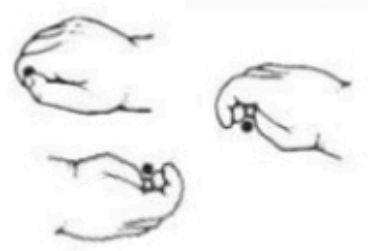

VARIATION IN MOVEMENT TYPE

e.g. used muscles

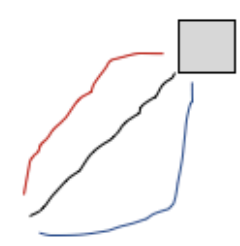

VARIATION IN TRAJECTORY e.g. combinations of muscles

Figure 1. Different manifestations of variability in motor output, here exemplified for reaching behavior

\section{Evidence that variable behaviour may reflect exploration}

Increased variability at key points in development. If variability is a nuisance then we'd expect development to proceed from more variable to less variable behavior. On the contrary, observing increasing variability, at certain moments in development, might point to it having a functional role. Motor activity starts early in fetal development. Fetal movements are varied and structured; rodents, for example, exhibit coordinated motor patterns antecedent to postnatal righting, locomotion, suckling, maternal-infant communication and grooming behavior (Robinson \& Smotherman, 1992). This activity decreases towards 40 weeks after gestation, whether the infant is born at term or pre-term, suggesting that this decrease does not reflect space limitation towards the end of the pregnancy but a pre-programmed pattern of up and down-regulating variability. This decrease in movement does not continue after term. Although the newborn must cope with the restraints of gravity, after birth there is an increase in the variability of writhing general movements, which are gross movements involving the 
whole body (Prechtl, 1993). These involve variable sequences of arm, leg, neck and trunk movements, with often slight changes in direction of the movement. In the author's own words: " These [...] make the movements fluent and elegant and create the impression of complexity and variability" (Prechtl, 1993).. An increase in combinatorial variability, in terms of a decrease in the locking of movement of different limbs is also observed from 6 to 18 weeks (Piek et al, 2002). It is unclear why increases in motor variability is observed at particular time points in the development but one suggestion has been that they reflect an increase in exploratory behavior accompanying new learning opportunities in development (Stulp \& Oudeyer, 2017). Sensitivity of variability to information availability may be a better way to tease apart wanted and unwanted variability. Some evidence for this is reviewed below.

Variability in atypical development. A decrease rather than an increase in the variability of motor outputs is observed in many pathologies of movement (e.g. Parkinson's Disease, Freund \& Hefter, 1993; stuttering, Grosjean, van Galen, de Jong, van Lieshout, \& Hulstijn, 1997) but also more generally whenever development is compromised. This is the case in infants with documented brain damage, who display monotonous and more stereotypical movements, less fluent and lacking complexity (Prechtl, 1993; Newell, van Emmerik, \& Sprague, 1993). Cerebral palsy has also been linked with decreased movement variability in the first few months of life (e.g., Prechtl, 1997). Vaal et al. (2000) showed that 18 and 26 weeks old infants with periventricular leukomalacia had tighter intra-limb locking during spontaneous kicking, compared with infants with no evidence of brain damage. Objectdirected behavior is also often reduced in atypical development. Nine-month-old high-risk preterm infants engaged in less fingering, rotation or transfer of objects and a summary exploration score predicted cognitive functioning at 24 months (Ruff et al, 1984). Preterm 
born infants showed decreased variability in reaching movements, both in terms of distal (e.g. reaching with both one or two hands) and proximal adjustments (e.g. various hand openings; Soares et al, 2014) and lower levels of exploratory movements of toys (Guimaraes et al, 2013; Soares et al, 2012). Variability in the execution of motor acts, as for example when standing up, seems to be the norm, with only a small number of 12-18 month olds showing very low, and therefore atypical, variability (Towen, 1993).

Of course, showing that certain pathologies associate with decreased motor variability is necessary but not sufficient evidence in support of the idea that increased variability is a driver of learning. Some pathologies are, such as Tourette's are associated with poor motor learning but increased variability (e.g. Draper et al., 2015). As is the case with changes in the amount of movement variability during development, direct evidence for an association between increased variability and information availability or acquisition, is needed.

Sensitivity to learning context. An increase in information availability has been shown to make infants increase motor activity or variability. When infants are engaged in reaching training regimes they produce distal adjustments that increase in variability (Soares et al, 2013). Across a number of habituation studies, introducing infants to an object with a new property increased object-directed movement and the variability of movement types. Steele \& Pederson, (1977) observed that 6-month-old infants increased their touching and looking behavior when introduced to an object that differed in temperature from previous ones, but no change in behavior occurred when the object changed colour. Nine to twelve-month olds engaged in more banging when exploring objects that had a new weight and more rotating and transferring when exploring objects that had a new shape (Ruff, 1984). When given an object with a new texture newborns increased the frequency of their hand pressure movements (Molina \& Jouen, 2004). In another study, information content modified infants 
mouthing of artificial nipples - more variable movements (and less sucking per se) was measured in newborns when they experienced a new nipple texture (Rochat, 1983). Rochat notes that this activity could not have been reflexive, since it was modulated in character and varied according to context.

Although this review is mainly concerned with early development, the principles under scrutiny here are not in any way restricted to infancy but will characterize any learning system, along lifespan. Thus, it was observed that vocal articulators increase in movement variability following cochlear implantation. The stability of movement trajectories for correctly produced speech was compared pre- and post-implantation, in a 7-year old child (Goffman, Ertmer and Erdle, 2002). Pre-implantation, the participant had slightly higher movement variability than age-matched controls. Two and four months after implantation, variability increased further but by six months post-implantation, this child produced speech movements of a similar stability as the controls. The authors comment that variable movements of correctly articulated speech 'may reflect a system that is being modified in response to new auditory input provided by the cochlear implant' (p. 892). The song of young male zebra finches increases in spectral variability when they sing in isolation, compared to when singing to a female (Kao \& Brainard, 2006). The authors suggested that males take advantage of solitary moments to explore vocal productions in view of improving their song. Although these studies are compatible with the idea that variation is upregulated to help learning, it still remains possible that an increase in variability in learning situations simply reflects imprecise motor control, that is failed attempts to achieve a new goal rather than exploration the best ways of achieving that goal. Showing that increasing variability actually promotes learning, provides the strongest evidence for its adaptive role in development or skill acquisition. This type of evidence remains scarce or indirect. 
Variability and learning. One of the most compelling studies leading variability in motor activity and learning, had adult participants learn a new motor routine. Wu et al (2014) measured variability of arm movements before and during a motor learning task in which participants had to draw subtly curved shapes, with fast arm movements. Variability during a baseline period (in which no feed-back was given for tracing a model curve), was positively correlated with how close to the target curve participants got in the training phase. It was variability in a task-relevant dimension that best predicted learning. McAllister et al., (2014) assessed the role of motor exploration for vocal learning and found that children enrolled in an ultrasound biofeedback intervention for $/ \mathrm{r} /$ mis-articulation only made progress when they were allowed to try out a variety of tongue shapes for $/ \mathrm{r} /$, rather than being set a specific shape by the therapist. More recently, Lee et al, (2017), showed worse learning of a new motor skill in children than adults. Participants had to use their upper body movements to control a cursor on a screen. The authors explained these findings based on children's limited exploration of their movement repertoire. Exploration was quantified here as the ratio between 2 principal components, emerging from PCA, and that explained most variance. This metric was considered to better capture exploration of the 2 dimensions of the screen than variation within each dimension.

No study yet has shown that progress in a particular learning task is improved in infants that manifest increased variability in behaviour (e.g. better discrimination of weight in infants that had manifested most variability in banging objects). We know, however, that motor maturity in infancy (e.g. whether infants can sit and reach) correlates with cognitive development in childhood (Bornstein, Hahn \& Suwalsky, 2013). Although this study did not measure variability in behavior per se, a child that is able to sit will be able to use their arms to explore a higher range of reaching and grasping strategies for objects of various shapes or 
sizes. Future studies will have to investigate whether the amount of motor variability is what mediates the association between early motor maturity and later cognitive development.

\section{The sources of behavioral variability}

One obvious source of variability in behaviour is the environment itself. However, this review is concerned with intrinsically generated variability. I will only refer to environmental variability when the child is responsible for actively creating it. So how could exploratory behaviour be generated? Gibson, (1988) suggested that infant exploratory activity "continues as play through the preschool years and as deliberate learning later in life", and this possibly reflects the view of many others. However, even for a gesture as simple as reaching, we have little evidence for developmental continuity between the mechanisms driving the various paths arm movements take when a 1-month-old explores reaching for an object, when a 12-month-old explores how to activate a new toy, or when, a year later, she figures out how to build a tower of blocks? It remains still possible that the balance between noise and exploratory behaviour, as sources of variability, shifts during development. This dichotomy between noise and exploration is also not that clear cut. In a recent review, Dhawale et al (2017) differentiate between planned noise, variability deliberately generated in the central nervous system and execution noise, variability resulting from the randomness of biological processes such as spike generation and propagation, synaptic transmission, muscle protein changes, in both the central or the peripheral nervous system. However, a closer look at the sources of planned noise or variability, reveals a spectrum of possible mechanisms. In its highest-level form, planned variability could be seen as a reflection of deliberate hypothesis testing. Children figuring out how to activate a hidden mechanism with the help of wooden blocks try various combinations of blocks and often verbalise the hypothesis they are testing (Gopnik et al., 2001). This process of hypothesis or theory testing 
is seen by some as critical for advancing learning, especially for generalizing knowledge beyond the particulars being experience at a moment in time (e.g. see Annette KarmiloffSmith's, "If you want to get ahead, get a theory"; Karmiloff-Smith \& Inhelder, 1974). How exactly hypotheses are generated remains largely unknown; in their study of balancing objects, Karmiloff-Smith \& Inhelder (1974) observed that past experience heavily influences which hypothesis children will try out (try balancing in the middle if that worked before), and formulating a new theory did not necessarily emerge from encountering counter-examples of the former theory, but from a process of insight, difficult to capture from children's behaviour.

There are however cases in which an individual does not have enough background knowledge to formulate explicit theories. To take a simple example, we might know where alternative sources of food could be if we don't find any in our fridge (try corner shop); but while in a forest and hungry, we might not even recognize what food looks like. Adopting quasi stochastic behaviour e.g. sampling anything that looks vaguely edible, might be our best bet in these situations. Rats faced with an unpredictable competitor for food, whose actions they try to counteract but fail, adopt a random pattern of choices between two food sources (Tervo et al., 2014). This change is mediated by noradrenergic input from the locus coeruleus into the anterior cingulate cortex (ACC), which suppresses ACC activity (i.e. the use of previous world models). In this case, despite randomness in the sequence of choices, behaviour still reflects discrete choices in a limited hypothesis space (the rat still only touches one of two potential locations of food). In other cases, though, variability in behaviour manifests as a normal distribution of choices, rather than discrete patterns. This is true of the adult song of Bengalese finches, in which song syllables are produced with a normal distribution of pitch values (Tumer \& Brainard, 2007). Arm reach angles of adult human participants learning a new motor task are also normally distributed (Pekny et al, 2015). 
However, despite the seemingly stochastic nature of this variability, we know that in both these cases variability does not result from noisy motor output, but is actively produced at the motor planning stage. Churchland et al., 2006 showed that even for highly practiced tasks, such as reaching, about half of the variability in monkey's reach speed originates in the premotor and motor cortex. The neural structures and physiological mechanisms through which pitch variability is produced in the finches' brain are also well known (Budzillo et al., 2017).

Is there evidence that this type of variability supports learning? The importance of finches' pitch variability for vocal learning is exemplified by Tumer \& Brainard (2007) work. By negatively reinforcing the upper end of a normal distribution of pitches through the contingent presentation of white noise, researchers succeed in shifting the pitch of a particular syllable in the song produced by finches. Interesting, the shift resulted in a distribution with a new mean, but which maintained the same degree of variability around the mean. Thus, this variability is actively maintained to enable the learning of new songs through reinforcement of particular ranges in the distribution, just like genetic variability is generated for natural selection to occur. I will call this learning expectant variability.

Finally, some variability in behavior will undoubtedly reflect motor noise. Could learning take advantage of this type variability at all? The reason this might be difficult is because the motor plan that was eventually executed is not the plan that was intended, and therefore a memory trace of the intended plan could not be reinforced. However, studies that have used passively generated variability suggest that sensory feed-back is sufficient for reinforcement learning to occur. For example, in Bernardi et al., (2015) adult participants learned a new motor contingency after only having been given passive exposure to a variety of trajectories to a particular target, some of which were reinforced as successful hits. Passive exposure was achieved by moving participant's limbs using a robot arm and resulted in the same learning success as active training. Thus, even in the absence of known motor plan, 
participants could discover successful motor sequences simply based on the sensory feedback they received from their limbs. However, in this case, recovery of the intended motor plans was made possible by known sensory-motor contingencies. Participants were adults who had a life time of experience with arm movements and therefore a fairly good idea of which of these movements could lead to the particular sensory feed-back. These assumptions will not hold at some point in infancy.

\section{Which of these mechanisms could generate exploratory behaviour in infancy?}

Where might infant exploratory behaviour be, on the continuum between hypothesis testing and sensory-motor noise (see Table 1)? More importantly, might there be something to gain from increased variability, whatever its source?

Hypothesis testing. Many have been captivated by the metaphor of infants as little scientists (see Alison Gopnik's “Scientist in the crib”), and indeed some early object exploration descriptions do seem compatible with primitive hypothesis testing. Infant's using different action patterns when reacting to changes in object properties (e.g. Steele \& Pederson, 1977; Ruff, 1984) could reflect deliberate testing of a "perceptual" hypothesis, e.g. banging might reflect infants' deliberate testing object weight and fingering, the optimal way of testing an object's temperature. These behaviors are very similar to the exploratory procedures used by adults when having to discriminate objects based on various properties (Lederman \& Klatzky, 1993). However, these behaviors need not reflect infants apriori appreciation that banging is a better way of learning about weight than about temperature. It may simply be that the unexpected change in temperature triggers exploratory behavior, just as unexpected environmental changes increase exploratory behavior in rats (e.g. Tervo et al., 2014). An increase in a variety of object directed actions (banging, fingering, mouthing) would, as a consequence, allow infants to discover that some of these actions bring about 
more information than others - e.g. that a new object's temperature is better perceived when fingering it. Fingering would therefore be gradually selected over other behaviors, in the process of infants interacting with objects but may not be stored in long term memory as the strategy to use for learning about temperature. Initially, infants would have to make this discovery at each encounter of a temperature change. Rather than hypothesis testing, this would, at least initially, reflect learning expectant variability.

Hypothesis testing was directly investigated in a recent study by Stahl \& Feigenson, 2015. Here, 12-month-olds manipulated objects differently following solidity versus support violations - they banged objects that had passed through walls, but dropped objects that had not obeyed gravity. In this case, the objects themselves did not give away any cues about their properties, which means infants must have apriori chosen which actions were best suited to test their previous observations. In another study, Needham \& Baillargeon, (2000) observed an intriguing association between the percentage of time $3 \frac{1}{2}$ month old infants looked at or mouthed objects they were holding and their ability to visually parse objects based on their surface features. While this might simply reflect that motorically advanced infants also have better visual processing skills, an alternative interpretation is that object manipulation, which involves breaking contact between objects, had helped infants formulate hypothesis about object structure, for example the hypothesis that discontinuity in surface features will result in objects being easily taken apart.

Is talking about hypothesis testing, in the above cases, too rich of an interpretation of infant's behaviour? In its simplest form, the hypotheses infants test involve acting on the world and expecting a particular outcome (e.g. when I bang this object, I will perceive its weight). But is it possible to demonstrate that infants build up specific expectations during exploration? In an EEG study in which infants could build specific expectation about learning either object functions or labels, theta-band activity was measured over frontal areas in 
anticipation of object functions, but temporal theta activity was measured when labels were expected (Begus, Gliga \& Southgate, 2016). Frontal theta band activity was measured also while infants explored objects (Begus, Southgate \& Gliga, 2015). These correlates of information expectation offer an opportunity to investigate the earlier forms on hypothesis testing driving infant object exploration.

Learning expectant variability. Is there evidence that infants produce the type of learning expectant variability that is the bread and butter of reinforcement learning? The increasing variability in reaching behaviour during the first year of life, may be a good candidate for this mechanism at play in infancy (Thelen, 1979; Prechtl, 1993). Infants given reaching and grasping practice, which includes reinforcement of successful reaches, increased the frequency of this behavior (Soares et al, 2013). Interestingly, training only increased grasping success in infants born at term (Soares et al., 2014), i.e. in those infants that showed higher variability in grasping behavior already before the intervention. This suggests that increased variability may give term infants more opportunities to discover optimal reaching strategies. However, only one published study reports on an attempt to directly reinforce a subset of the spatial positions that 5-month-old infants' hands took during reaching (Darcheville, Boyer \& Miossec, 2004), a manipulation similar to the reinforcement of particular pitches in zebra finches' song. In this study, the arrival of infant's hand within particular spatial positions was automatically detected and generated a recording of mother's voice. This manipulation increased reaching behavior; we do not know, however, whether this was accompanied by an increase in reaching using the reinforced trajectory. Selective reinforcement of either consonants and vowels (through smiling, vocal responses and touch) works to increase infants' production of these phoneme classes (Routh, 1969). There is some evidence that mothers themselves selectively reinforce infant vocalizations, as for example imitating infant consonant production more than vowel productions (Gros-Louis et al, 2006). 
Again, evidence for reinforcement of vocal behavior also falls short of telling us whether learning takes advantage of the increased variability in infants' vocal productions, for example.

Environmental variability. Reinforcement learning is central to computational models of reaching (Caligiore, Parisi \& Baldassarre, 2014) and vocal development (Mouin-Fryer \& Oudeyer, 2012) and these models critically depend on an initial pool of variable behavior. To model reaching development, Caligiore, Parisi \& Baldassarre (2014) used what they call exploratory noise, i.e. random perturbations in the motor output, to which muscular noise is added. Interestingly, these authors suggest that much of the exploratory noise is not actually planned by the child herself, but is a consequence of the child interacting in with her (unpredictable) environment. For example, when reaching for an object, another object might block her way and change the reaching trajectory; reaching might change her center of gravity and this in turn could affect the trajectory her arm takes towards an object. Again, the question to ask here is to what extent the child seeks to actively increase environmental variability through her actions. Fagan \& Iverson (2007) suggested that one of the functions served by object mouthing, during early play, is to add variability to vocal output - some kind of lucky accidents; they went on to show that a larger variety of glottal and sub-glottal sounds were produced when infants were vocalizing while mouthing objects. However, none of the sounds produced were new, in the sense that they were well in the repertoire of an infant that age. Mechanistically, this type of variability is not different from variability produced by noisy motor outputs, since the child is not in control of it, i.e. not in possession of the motor plans that yielded the final behavior. A priori, these motor plans could still be retrieved by making use of the sensory output of these actions and mapping them back on the closest motor plan(s). Of course, had the sound produced been a new sound, a corresponding motor plan would not exist. This strategy of increasing vocal variability is therefore unlikely 
to be a driver phonological development. The best a learner can do, if a new sound results from them mouthing objects, is to access the nearest motor plan available, the motor plan corresponding to the closest sound in their repertoire. Added variability to the closest available motor plan may just take the infant close to the new sound they aimed for. Moreover, given young infants poor memory, sifting through these motor plans should occur fast enough, before she forgets the new sound she produced and wants to re-enact. The solution to that is one other feature of early exploratory activity. In addition to being highly variable, exploration is also highly repetitive.

The role of repetitive behaviour. Repetitive patterns of behaviour are present in both limb and vocal movement, and in higher frequency at particular time points in development. Repetitive actions with objects are present at high frequency during infancy, being ubiquitous at 12 months (Fyfield, 2014). Repetitions per vocalization increase and peak around 9.5 months (Fagan, 2009) but decline with word production (only 18\% of first words contain 2 reduplicated syllables Vihman, 1996). With increase motor control, infants could actually produce more reduplication, but they do not. Thus, the amount of reduplication does not reflect competence, but seems to serve a particular function during particular windows of development. I suggest here that this type of repetitive behaviour serves the same function as the learning expectant variability described in finches. A detailed analysis of reduplicated behaviour will reveal that repetitions are not identical; generating a train of actions, rather than one, ensures that enough variability is produced in a short period of time. We can see how this helps in the case in which new motor effects are discovered opportunistically, as when mouthing objects. I predict that the production of new sounds, when mouthing objects, will be followed by an increase in both vocal output and reduplications; the higher the vocal output/reduplications, the higher the chance or re-enacting the newly produced sound, even in 
the absence of objects. This hypothesis is supported by evidence that repetitive behaviour is modulated by contextual variables, such as the availability of information. Cyclical grasping can be elicited in 3-day-old infants when they are handed objects with new textures (Molina \& Jouen, 2004). Reduplicate babbling is also enhanced when auditory feed-back is received: deaf infants show delayed or absent reduplication (Oller \& Eilers, 1988; Koopmans-van Beinum, Clement, \& van den Dikkenberg-Pot, 2001). Reduplication does appear in vocal production weeks after cochlear implants and, interestingly, precedes an increase in the quality of the consonant vowel vocalizations themselves (Fagan, 2015).

\section{Play and exploration}

A distinction was made between exploration and play, which I have reformulated as a distinction between discovering means to achieve a particular goal (in exploration) and generating new goals (in play). More precisely, these new goals are a step ahead from the ones previously explored. This latter perspective on play is close to Vygotsky's: "In play it is as though he were a head taller than himself" (Vygotsky, 1930-1935/1978, p.102). A tight dynamic might exist between play and exploration, where the latter describes behaviour that allows children to embark in new explorations once they have exhausted previous exploratory opportunities (e.g. exploring how to grasp a very slippery object vs. trying to grasp the object with one's foot). Through this perspective, some of the behaviour described in this review might well fit with the definition of play, as for example when mouthing objects leads to producing new sounds.

\section{Conclusion}

We set to answer the question of whether the "noisiness" and variability of behavior in infant early behavior is actively generated, rather than being the signature of an immature 
motor system. Evidence for contextual modulation of motor variability, especially evidence that variability increases with information availability, and a better understanding of the neural sources of variability, suggests that, even early in development, variability might be upregulated in support of learning. However, strong support for this hypothesis still awaits a better characterisation of infant motor variability per se, in the same way in which it has been characterised in bird vocal learning or adult motor skill acquisition. A better characterization of motor outputs and their contextual modulation will allow us to understand to what extent they reflect hypothesis testing, learning expectant variability, or merely lucky accidents.

\begin{tabular}{|l|l|l|}
\hline Sources of variability in behavior & Linked to learning & Present during infancy \\
\hline Hypothesis testing & yes & Stahl \& Feigenson, 2015 \\
\hline Learning expectant variability & yes & Soares et al., 2014 \\
\hline Environmental variability & yes & Fagan \& Iverson (2007) \\
\hline Sensory-motor noise & unclear & certainly \\
\hline
\end{tabular}

Table 1. Potential sources of variable behaviour in early play

Acknowledgments

I wish to thank the UK Medical Research Council (G0701484), who funded me while writing this article. Also, thanks to Clare Press for commenting on an earlier draft of this manuscript. 


\section{References}

Begus, K., Southgate, V., \& Gliga, T. (2015). Neural mechanisms of infant learning: differences in frontal theta activity during object exploration modulate subsequent object recognition. Biology letters, 11(5), 20150041.

Begus, K., Gliga, T., \& Southgate, V. (2016). Infants' preferences for native speakers are associated with an expectation of information. Proceedings of the National Academy of Sciences, 113(44), 12397-12402.

Bernardi, N. F., Darainy, M., \& Ostry, D. J. (2015). Somatosensory contribution to the initial stages of human motor learning. Journal of neuroscience, 35(42), 14316-14326.

Blumberg, M.S, Coleman CM, Gerth AI, McMurray B. Spatiotemporal structure of REM sleep twitching reveals developmental origins of motor synergies. Current Biology. 2013;23:2100-2109.

Bonawitz, E., Shafto, P., Gweon, H., Goodman, N. D., Spelke, E., \& Schulz, L. (2011). The double-edged sword of pedagogy: Instruction limits spontaneous exploration and discovery. Cognition, 120(3), 322-330.

Bornstein, M. H., Hahn, C. S., \& Suwalsky, J. T. (2013). Physically developed and exploratory young infants contribute to their own long-term academic achievement. Psychological science, 24(10), 1906-1917.

Budzillo, A., Duffy, A., Miller, K. E., Fairhall, A. L., \& Perkel, D. J. (2017). Dopaminergic modulation of basal ganglia output through coupled excitation-inhibition. Proceedings of the National Academy of Sciences, 114(22), 5713-5718.

Burghardt, G.M. (2014). A brief glimpse at the long evolutionary history of play. Anim. Behav. Cogn. 1, 90-98.

Caligiore, D., Parisi, D., \& Baldassarre, G. (2014). Integrating reinforcement learning, equilibrium points, and minimum variance to understand the development of reaching: a computational model. Psychological review, 121(3), 389.

Churchland, M. M., Afshar, A., \& Shenoy, K. V. (2006). A central source of movement variability. Neuron, 52(6), 1085-1096.

Dhawale, A. K., Smith, M. A., \& Ölveczky, B. P. (2017). The Role of Variability in Motor Learning. Annual Review of Neuroscience, (0).

DiPietro, J. A., Bornstein, M. H., Costigan, K. A., Pressman, E. K., Hahn, C. S., Painter, K., ... \& Yi, L. J. (2002). What does fetal movement predict about behavior during the first two years of life?. Developmental psychobiology, 40(4), 358-371. 
Draper, A., Jude, L., Jackson, G. M., \& Jackson, S. R. (2015). Motor excitability during movement preparation in Tourette syndrome. Journal of neuropsychology, 9(1), 33-44.

Fagan, M. K. (2015). Why repetition? Repetitive babbling, auditory feedback, and cochlear implantation. Journal of experimental child psychology, 137, 125-136.

Fagan, M. K. (2009). Mean Length of Utterance before words and grammar: Longitudinal trends and developmental implications of infant vocalizations. Journal of child language, 36(3), 495-527.

Fagan MK, Iverson JM. The influence of mouthing on infant vocalization. Infancy 2007;11:191-202.

Ferguson, C. A., \& Macken, M. A. (1983). The role of play in phonological development. Children's language, 4, 231-254.

Freund, H. J., \& Hefter, H. (1993). The role of basal ganglia in rhythmic movement. Advances in neurology, 60, 88 .

Fyfield, R. (2014). The rise and fall of repetitive behaviours in a community sample of infants and toddlers (Doctoral dissertation, Cardiff University).

Gibson, E.J. (1988). Exploratory behavior in the development of perceiving, acting, and the acquiring of knowledge. Annual Review Psychology, 39, 1-41

Gliga, T., Jones, E. J., \& Johnson, M. H. (2015). Low noise in autism: Cause or consequence?. Autism, 19(3), 369-370.

Gliga, T., Smith, T. J., Likely, N., Charman, T., \& Johnson, M. H. (2015). Early visual foraging in relationship to familial risk for autism and hyperactivity/inattention. Journal of attention disorders, 1087054715616490.

Goffman, L., Ertmer, D. J., \& Erdle, C. (2002). Changes in speech production in a child with a cochlear implant: acoustic and kinematic evidence. Journal of Speech, Language \& Hearing Research, 45(5).

Gopnik, A., Sobel, D. M., Schulz, L. E., \& Glymour, C. (2001). Causal learning mechanisms in very young children: two-, three-, and four-year-olds infer causal relations from patterns of variation and covariation. Developmental psychology, 37(5), 620.

Gros-Louis, J., West, M. J., Goldstein, M. H., \& King, A. P. (2006). Mothers provide differential feedback to infants' prelinguistic sounds. International Journal of Behavioral Development, 30(6), 509-516.

Grosjean, M., van Galen, G. P., de Jong, P., van Lieshout, P. H., \& Hulstijn, W. (1997). Is stuttering caused by failing neuromuscular force control? In Speech production: Motor control, brain research and fluency disorders (pp. 197-204). Elsevier Science. 
Guimarães, E. L., Cunha, A. B., Soares, D. D. A., \& Tudella, E. (2013). Reaching behavior in preterm infants during the first year of life: A systematic review. Motor control, 17(4), 340354.

Harris, C. M., \& Wolpert, D. M. (1998). Signal-dependent noise determines motor planning. Nature, 394(6695), 780-784.

Inácio, A. R., Nasretdinov, A., Lebedeva, J., \& Khazipov, R. (2016). Sensory feedback synchronizes motor and sensory neuronal networks in the neonatal rat spinal cord. Nature communications, 7, 13060.

Izawa, J., \& Shadmehr, R. (2011). Learning from sensory and reward prediction errors during motor adaptation. PLoS computational biology, 7(3), e1002012.

Karmiloff-Smith, A., \& Inhelder, B. (1975). If you want to get ahead, get a theory. Cognition, 3(3), 195-212.

Kao, M. H., \& Brainard, M. S. (2006). Lesions of an avian basal ganglia circuit prevent context-dependent changes to song variability. Journal of neurophysiology, 96(3), 14411455 .

Karlsson, M. P., Tervo, D. G., \& Karpova, A. Y. (2012). Network resets in medial prefrontal cortex mark the onset of behavioral uncertainty. Science, 338(6103), 135-139.

Khazipov, R. et al. Early motor activity drives spindle bursts in the developing somatosensory cortex. Nature 432, 758-761 (2004).

Koopmans-van Beinum, F. J., Clement, C. J., \& Den Dikkenberg-Pot, V. (2001). Babbling and the lack of auditory speech perception: a matter of coordination? Developmental Science, 4(1), 61-70.

Lederman, S. J., \& Klatzky, R. L. (1993). Extracting object properties through haptic exploration. Acta psychologica, 84(1), 29-40.

Lee, M. H., Farshchiansadegh, A., \& Ranganathan, R. (2017). Children show limited movement repertoire when learning a novel motor skill. Developmental Science. Leekam, S. R., Prior, M. R., \& Uljarevic, M. (2011). Restricted and repetitive behaviors in autism spectrum disorders: a review of research in the last decade. Psychological bulletin, 137(4), 562.

Markant, D. B., \& Gureckis, T. M. (2014). Is it better to select or to receive? Learning via active and passive hypothesis testing. Journal of Experimental Psychology: General, 143(1), 94. 
McAllister Byun, T., Hitchcock, E. R., \& Swartz, M. T. (2014). Retroflex versus bunched in treatment for /r/ misarticulation: Evidence from ultrasound biofeedback intervention. Journal of Speech, Language, and Hearing Research, 57(6), 2116-2130.

Milh, M. et al. Rapid cortical oscillations and early motor activity in premature human neonate. Cereb. Cortex. 17, 1582-1594 (2007).

Molina, M., \& Jouen, F. (2004). Manual cyclical activity as an exploratory tool in neonates. Infant Behavior and Development, 27(1), 42-53.

Moulin-Frier, C., \& Oudeyer, P. Y. (2012). Curiosity-driven phonetic learning.

In Development and Learning and Epigenetic Robotics (ICDL), 2012 IEEE International Conference on (pp. 1-8). IEEE.

Needham, A., \& Baillargeon, R. (2000). Infants' use of featural and experiential information in segregating and individuating objects: a reply to. Cognition, 74(3), 255-284.

Oller, D. K., \& Eilers, R. E. (1988). The role of audition in infant babbling. Child development, 441-449.

Oudeyer, P. Y., \& Kaplan, F. (2009). What is intrinsic motivation? A typology of computational approaches. Frontiers in neurorobotics, 1, 6.

Pekny, S. E., Izawa, J., \& Shadmehr, R. (2015). Reward-dependent modulation of movement variability. Journal of Neuroscience, 35(9), 4015-4024.

Piek, J. P., Gasson, N., Barrett, N., \& Case, I. (2002). Limb and gender differences in the development of coordination in early infancy. Human movement science, 21(5), 621-639. Pierce, K., \& Courchesne, E. (2001). Evidence for a cerebellar role in reduced exploration and stereotyped behavior in autism. Biological psychiatry, 49(8), 655-664.

Prechtl, H. F. (1993). Principles of early motor development in the human. Motor development in early and later childhood: Longitudinal approaches, 35-50.

Prechtl, H. F. (1997). State of the art of a new functional assessment of the young nervous system. An early predictor of cerebral palsy. Early human development, 50(1), 1-11. Robinson, S. R., \& Smotherman, W. P. (1992). Fundamental motor patterns of the mammalian fetus. Developmental Neurobiology, 23(10), 1574-1600.

Robinson, S. R., Blumberg, M. S., Lane, M. S. \& Kreber, L. A. Spontaneous motor activity in fetal and infant rats is organized into discrete multi-limb bouts. Behav.

Neurosci. 114, 328-336 (2000).

Rochat, P. (1983). Oral touch in young infants: Response to variations of nipple characteristics in the first months of life. International Journal of Behavioral Development, 6(2), 123-133. 
Rubin, K.H., Fein, G.G. \& Vandenberg, B. (1983) Play. In P.H. Mussen \& E.M.

Hetherington (Eds.), Handbook of child Psychology (Vol. 4, pp 693-774). New York: Wiley. Ruff, H. A. (1984). Infants' manipulative exploration of objects: Effects of age and object characteristics. Developmental Psychology, 20(1), 9.

Soares, D., von Hofsten, C., \& Tudella, E. (2012). Development of exploratory behavior in late preterm infants. Infant Behavior and Development, 35(4), 912-915.

Soares, D. A., van der Kamp, J., Savelsbergh, G. J., \& Tudella, E. (2013). The effect of a short bout of practice on reaching behavior in late preterm infants at the onset of reaching: A randomized controlled trial. Research in Developmental Disabilities, 34, 4546-4558.

Soares, D., Cunha, A. B., \& Tudella, E. (2014). Differences between late preterm and fullterm infants: comparing effects of a short bout of practice on early reaching behavior. Research in developmental disabilities, 35(11), 3096-3107.

Stahl, A. E., \& Feigenson, L. (2015). Observing the unexpected enhances infants' learning and exploration. Science, 348(6230), 91-94.

Steele, D., \& Pederson, D. R. (1977). Stimulus variables which affect the concordance of visual and manipulative exploration in six-month-old infants. Child Development, 104-111. Swanson, M. R., Shen, M. D., Wolff, J. J., Boyd, B., Clements, M., Rehg, J., ... \& Hazlett, H. C. (2017). Naturalistic Language Recordings Reveal "Hypervocal” Infants at High Familial Risk for Autism. Child Development.

Tervo, D. G., Proskurin, M., Manakov, M., Kabra, M., Vollmer, A., Branson, K., \& Karpova, A. Y. (2014). Behavioral variability through stochastic choice and its gating by anterior cingulate cortex. Cell, 159(1), 21-32.

Thelen, E. (1979). Rhythmical stereotypies in normal human infants. Animal behaviour, 27, 699-715.

Thelen, E., \& Corbetta, D. (1994). Exploration and selection in the early acquisition of skill. International Review of Neurobiology, 75-75.

Todorov, E., \& Jordan, M. I. (2002). Optimal feedback control as a theory of motor coordination. Nature neuroscience, 5(11), 1226-1235.

Towen, B.C. (1993) How normal is variable, or how variable is normal ? Early human development, $34,1-12$

Tumer, E. C., \& Brainard, M. S. (2007). Performance variability enables adaptive plasticity of 'crystallized'adult birdsong. Nature, 450(7173), 1240-1244. 
Vaal, J., van Soest, A. K., Hopkins, B., \& Sie, L. T. (2002). Spontaneous leg movements in infants with and without periventricular leukomalacia: effects of unilateral weighting. Behavioural brain research, 129(1), 83-92.

Wilcox, T. (1999). Object individuation: Infants' use of shape, size, pattern, and color. Cognition, 72(2), 125-166.

Woolley, S. C., \& Doupe, A. J. (2008). Social context-induced song variation affects female behavior and gene expression. PLoS biology, 6(3), e62.

Wu, H. G., Miyamoto, Y. R., Castro, L. N. G., Ölveczky, B. P., \& Smith, M. A. (2014).

Temporal structure of motor variability is dynamically regulated and predicts motor learning ability. Nature neuroscience, 17(2), 312-321.

Yan, J. H., Thomas, J. R., Stelmach, G. E., \& Thomas, K. T. (2000). Developmental features of rapid aiming arm movements across the lifespan. Journal of motor behavior, 32(2), 121140.

Yirmiya, N., Gamliel, I., Shaked, M., \& Sigman, M. (2007). Cognitive and verbal abilities of 24-to 36-month-old siblings of children with autism. Journal of autism and developmental disorders, 37(2), 218-229. 